\title{
BMJ Open Testing the information-motivation- behavioural skills model of diabetes self-management among Chinese adults with type 2 diabetes: a protocol of a 3-month follow-up study
}

Tingting Liu, ${ }^{1}$ Dongmei Wu, ${ }^{2}$ Jing Wang, ${ }^{3}$ Changwei $\mathrm{Li},{ }^{4}$ Rumei Yang, ${ }^{5}$ Song Ge,${ }^{6}$ Yan Du, ${ }^{7}$ Yanyan Wang ${ }^{8}$

To cite: Liu T, Wu D, Wang J, et al. Testing the informationmotivation-behavioural skills model of diabetes selfmanagement among Chinese adults with type 2 diabetes: a protocol of a 3-month follow-up study. BMJ Open 2018;8:e020894. doi:10.1136/ bmjopen-2017-020894

- Prepublication history for this paper is available online. To view these files, please visit the journal online (http://dx.doi. org/10.1136/bmjopen-2017020894).

TL and DW contributed equally.

Received 4 December 2017

Revised 2 June 2018

Accepted 6 June 2018
D) Check for updates

(C) Author(s) (or their employer(s)) 2018. Re-use permitted under CC BY-NC. No commercial re-use. See rights and permissions. Published by BMJ.

For numbered affiliations see end of article.

Correspondence to

Dr Tingting Liu; tl023@uark.edu

\section{ABSTRACT}

Introduction Currently, China leads the world in the number of people with diabetes, making it home to a third of the global diabetic population. Persons with diabetes have to carry out $95 \%$ of their self-care. As an important component of diabetes care, diabetes selfmanagement (DSM) is defined as everyday behaviours that persons carry out to control diabetes. Consistent findings have been reported that level of compliance to suggested DSM behaviours is not considered optimal among Chinese adults with type 2 diabetes (T2D). The underlying reasons for suboptimal DSM behaviours among Chinese adults are not well known and no conceptual model has been developed to guide DSM interventions in this population. Although the information-motivation-behavioural skills model has been tested among Chinese adults with T2D, some key components of the original model were not tested. In this proposed study protocol, we will refine and test a culturally tailored model of DSM longitudinally among 250 Chinese adults residing in China.

Methods and analysis This is a descriptive, repeated-measure study to be conducted at a tertiary hospital in Chengdu, China. A total of 250 adults with T2D will be enrolled and followed for 3 months in this study. Information of multiple domains will be collected, including demographics, diabetes knowledge, health education form, provider-patient communication, health beliefs, social support, diabetes self-efficacy, the medical coping modes, the diabetes self-care, depression, diabetes-dependent quality of life, haemoglobin A1c, blood pressure and blood lipids at baseline and 3-month follow-up. Main analyses comprise linear regression modelling controlling for covariates and structural equation modelling.

Ethics and dissemination Ethical approval has been obtained through the Fourth People's Hospital of Chengdu Research Ethics Committee (study approval number 2017017). We aim to disseminate the findings through international conferences, international peer-reviewed journals and social media.

Trial registration number ChiCTR-R0C-17013592.

\section{Strengths and limitations of this study}

- This study will recruit and follow-up Chinese adults with diabetes for 3 months, and test the information-motivation-behavioural skills model of diabetes self-management longitudinally for the first time.

- This study relies primarily on self-reported measures and the objective, observable levels of diabetes self-care behaviours that should be used in future studies

- The study will be conducted in Chengdu, a city in South-Western China, and therefore the findings of the study may not be generalised to other parts of China, and the chosen centres are not from areas that are representative of the general Chinese population with regards to socioeconomic status.

- This is a descriptive, repeated-measure study. A randomised-controlled trial with a group of patients receiving no DSM interventions would have been desirable to test the model.

\section{INTRODUCTION}

Diabetes is a global public health challenge because of its high prevalence and associated mortality and morbidity. The estimated number of individuals with diabetes is estimated to increase from 425 million in 2017 to 649 million in 2045 worldwide, with over $75 \%$ of people with diabetes residing in low-income and middle-income countries. ${ }^{1}$ The most comprehensive nationwide survey showed that approximately 114 million $(11.6 \%)$ Chinese adults in total had diabetes in 2010, and this was a twofold rise over the past decade. $^{2}$ About $90 \%-95 \%$ of people with diabetes have type 2 diabetes (T2D). ${ }^{3}$ Individuals with T2D carry out $95 \%$ of their diabetes care. ${ }^{4}$ Diabetes self-management (DSM) is therefore an important part of diabetes care, and is defined as daily behaviours that 
persons carry out to control T2D, including self-monitoring blood glucose (SMBG), dietary changes, engaging in regular physical activity, diabetes foot care, managing high or low blood glucose, taking prescribed medications and smoking cessation. ${ }^{5}{ }^{6}$ DSM is complex, and involves major lifestyle changes which need to become a part of a person's daily routine and require high levels of adherence to these lifestyle changes. ${ }^{7}$

Mounting evidence has consistently demonstrated that better DSM was associated with improved health outcomes, including lower haemoglobin A1c (HbA1c) levels, ${ }^{8}$ better quality of life $^{9}$ and decreased incidence of diabetes-related complications. ${ }^{10}$ It has been reported that persons with diabetes can make a great impact on the progression of diabetes by performing their own care. However, adherence to some of DSM behaviours has been found to be low, especially when looking at longterm behavioural changes. ${ }^{11}$ This suggests a critical need for a comprehensive and well-tested conceptual model to guide future DSM interventions.

Although the information-motivation-behavioural (IMB) skills model has been tested among Chinese adults with $\mathrm{T} 2 \mathrm{D},{ }^{12}$ some key components of the original IMB model were not tested. For example, it is well known that personal motivation, such as health beliefs, may influence DSM among Chinese adults. ${ }^{13}$ Additionally, important moderators known to influence DSM in Chinese adults such as depressive symptoms, female gender and educational level were not included in the model, ${ }^{12}$ and this may add important insights into DSM specific to this population. Therefore, a revised conceptual model based on the IMB model is needed to take into account the effects of the interactive relationships among these related factors. The proposed IMB-DSM model will help fill this gap by examining the potential moderators of DSM in this population, so that intervention studies based on this model will show whether the intervention works, and why it works and under what conditions it works.

\section{Conceptual framework}

Overview of the IMB model

IMB provides the theoretical basis for this study. The IMB model is used to frame these variables because: (A) The concepts from the IMB model can easily be translated into intervention components. ${ }^{14}$ (B) The IMB model adequately captures essential concepts which have been well supported in the literature to improve DSM behaviours, including information (eg, diabetes knowledge), motivation (eg, social support) and behavioural skills (eg, diabetes self-efficacy). The model postulates that individuals are more likely to take health-related actions, such as DSM behaviours, if they are well informed, highly motivated (personally and socially), and have adequate and appropriate behaviours, and thus, experience positive health outcomes. ${ }^{15}$ The model is based on three constructs: information, motivation and behavioural skills. For behaviours that are complex such as DSM, information and motivation are thought to work largely through behavioural skills to initiate and maintain the behaviour at focus, and the behaviour is directly linked to health outcomes, which, in turn, are believed to affect one's maintenance of the behaviour through a feedback loop that influence one's future levels of IMB skills over time. ${ }^{16}{ }^{17}$ The IMB model further postulates that favourable health outcomes may strengthen an individual's IMB skills to perform the behaviour in future. In contrast, unfavourable health outcomes may cause an individual to lose confidence in his or her knowledge, motivation and behavioural skills to perform the behaviour over time. ${ }^{17}$

\section{Overview of the IMB-DSM model}

Figure 1 portrays the overarching conceptual framework of this study. The IMB-DSM model provides comprehensive insight into the relationships that affect DSM and its related health outcomes, based on a systematic review of literature in this area. The framework consists of the following six key interrelated components: DSM-related information, DSM-related motivation, DSM-related behavioural skills, DSM behaviours, health outcomes and moderators. Consistent with the original IMB assumptions, the IMB-DSM model asserts that DSM-related IMB skills are fundamental determinants of DSM behaviours. DSM-related information and motivation are proposed to work primarily through behavioural skills to affect the initiation and maintenance of DSM behaviours. DSM behaviours lead to health outcomes, which, in turn, can influence one's future maintenance of the behaviour through a feedback loop that affects one's levels of DSM-related IMB skills over time. The proposed moderators can influence the relationship among the variables in the IMB-DSM model. Each part of the IMB-DSM model is presented in detail below.

\section{DSM-related information}

DSM-related information is a necessary but insufficient prerequisite for DSM behaviours. The link between DSM-related information and DSM behaviours has been well established in the literature. DSM-related information includes knowledge of diabetes, health education and provider-patient communication. A positive relationship was found between knowledge of diabetes and overall DSM performance, ${ }^{18-20}$ exercise, foot care, ${ }^{21}$ diet control, or $\mathrm{SMBG}^{22}$ in some studies, but not others. ${ }^{23} 24$ A significantly positive relationship was reported between health education, overall DSM performance ${ }^{25-27}$ and some specific DSM behaviours, such as diet modification, foot care, managing high or low blood glucose, SMBG, exercising, taking prescribed medications, smoking cessation. ${ }^{25} 2829$ Three studies examined the relationship between provider-patient communication and DSM behaviours, and found that better provider-patient communication was an independent, direct predictor of better overall DSM performance. ${ }^{12} 2430$ 


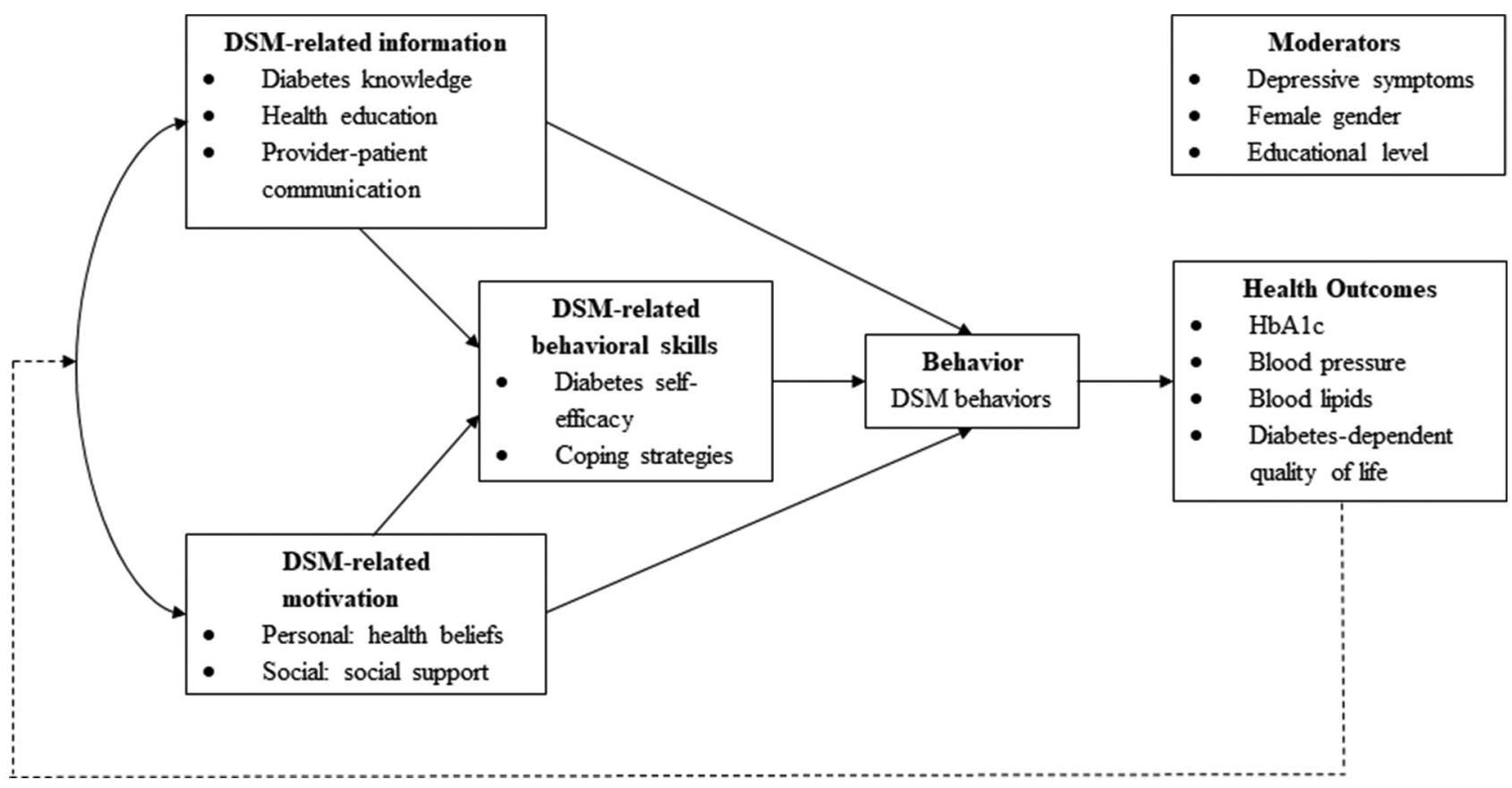

Figure 1 The DSM-IMB model. DSM, diabetes self-management; HbA1c, haemoglobin A1c; IMB, informatiom-motivationbehaviour.

\section{DSM-related motivation}

Another fundamental determinant of DSM behaviours is an individual's motivation to perform DSM. Consistent with the IMB model, the IMB-DSM model posits that an individual's motivation includes personal and social motivation. Personal motivation to perform DSM refers to one's beliefs about the DSM behaviours and evaluations of the outcomes. Social motivation to perform DSM refers to one's perception about social support for DSM and one's motivation to adhere to important others. ${ }^{31}$

In the IMB-DSM model, health beliefs are used to conceptualise personal motivation. Perceived susceptibility, perceived barriers, perceived benefits, or cues to action have each been related to overall DSM performance and all DSM behaviours except smoking cessation positively. ${ }^{1332}$ There was inconsistent evidence on the relationship between perceived severity and DSM behaviours. $\mathrm{Yu}^{13}$ reported a negative association, whereas Sun and co-workers ${ }^{11}$ reported a positive association. One possible explanation for the inconsistency between perceived severity and DSM behaviours involves the fact that both studies are cross-sectional. Thus, a person who is currently engaging in DSM behaviours may perceive himself or herself as not being at risk and may report few feelings of severity. Alternatively, a person who is presently engaging in less DSM behaviours may report more feelings of severity and few risk-reduction efforts. The inconsistency warrants further investigations from longitudinal studies. Consistent findings have been reported between higher perceived social support and better overall DSM performance, diet control, taking medications, taking regular exercise, SMBG, diabetes foot care, and managing high or low blood glucose. ${ }^{12} 212433-35$

\section{DSM-related behavioural skills}

Behavioural skills involve objective and perceived skills for performing DSM behaviours and a feeling of confidence for doing so. ${ }^{31}$ In the IMB-DSM model, behavioural skills include diabetes self-efficacy and positive coping strategy. A large number of studies have reported a consistently strong association between levels of self-efficacy and DSM behaviours, including diet modification, taking medications, foot care, taking regular exercise, SMBG, and managing high or low blood glucose. ${ }^{12} 1921243436-39$ Coping strategies have been well studied in this population. Research evidence is consistent with a positive relationship between confrontation and overall DSM performance, ${ }^{40}$ dietary modification, taking medications, foot care, physical activity, SMBG, or managing high or low blood glucose. ${ }^{13} 3236$ Alternatively, acceptance-resignation was negatively associated with overall DSM performance, ${ }^{40}$ dietary modification, taking medications, foot care, physical activity, SMBG, or regulating highs and lows in blood glucose. ${ }^{13236}$ Avoidance had negative effects on taking medications ${ }^{132}$ and regulating highs and lows in blood glucose. ${ }^{32}$

One cross-sectional study supported the role of DSM-related behavioural skills as a mediator between DSM-related information, motivation and DSM behaviours. Data on demographics, provider-patient communication, social support and self-efficacy, and diabetes selfcare were collected among 222 participants with T2D in Shanghai. There were significant positive and direct paths from self-efficacy $(\beta=0.41, \mathrm{p}<0.001)$, social support $(\beta=0.19, \quad \mathrm{P}=0.007)$ and provider-patient communication $(\beta=0.12, p=0.037)$ to DSM behaviours. Paths from provider-patient communication to self-efficacy $(\beta=0.23$, 
$\mathrm{p}<0.001)$ and from social support to self-efficacy $(\beta=0.19$, $\mathrm{p}<0.05)$ were significant and positive. Structural equations modelling showed that self-efficacy mediated the effects of social support (indirect effect $\beta=0.08, p=0.008$ ) and provider-patient communication (indirect effect $\beta=0.09, p=0.002$ ) on DSM behaviours. ${ }^{12}$ Overall, the study provided support for the specific direct and mediating relationships between DSM-related IMB skills and DSM behaviours. However, one limitation of this study is that the adapted IMB model does not include all relevant constructs. For example, information on personal motivation was not collected. Therefore, the study only partially tested the IMB model.

\section{DSM behaviours}

DSM behaviours primarily include dietary changes, weight loss and increased physical activity, SMBG, foot care and taking prescribed medications. Based on the guidelines of the American Diabetes Association, ${ }^{10}$ individuals with $\mathrm{T} 2 \mathrm{D}$ are encouraged to increase intake of whole grains, fibre, vegetables and fruits, and to reduce intake of total and saturated fat, sugar-flavoured beverages and high-calorie snacks. They are also encouraged to engage in $150 \mathrm{~min}$ or more of moderate-intensity to vigorous-intensity physical activity per week, and to lose about $5 \%-7 \%$ of initial body weight if they are overweight/obese.

\section{Health outcomes}

The IMB-DSM model asserts that DSM behaviours are directly linked to health outcomes, which has been well supported in the literature. Since adults with T2D are two to four times more likely to have cardiovascular disease (CVD) than adults without T2D, ${ }^{10}$ monitoring cardiometabolic markers is essential in the clinical management of patients with T2D. In the proposed study, blood pressure and blood lipids are measured to identify subsequent CVD risks among adults with T2D. The health outcomes include HbA1c, blood pressure, blood lipids and diabetes-dependent quality of life. DSM behaviour was directly related to glycaemic control $(\beta=-0.02, p=0.007),{ }^{30}$ total cholesterol $(\mathrm{TC}) /$ high-density lipoprotein cholesterol (HDL-C) $(\beta=-.31, p<0.001)$ and low-density lipoprotein cholesterol (LDL-C) / HDL-C $(\beta=-.30, p<0.001) .{ }^{12}$ It is not clear whether DSM behaviours are directly related to blood pressure in Chinese adults with T2D; this will be examined in this proposed study. Compared with the general population, people affected by T2D consistently reported diminished health-related quality of life. ${ }^{41}$ However, the association of DSM behaviours with health-related quality of life has not been systematically examined among Chinese adults with T2D, and this study will fill the knowledge gaps.

\section{Moderators}

The IMB-DSM model postulates that certain personal characteristics may work as moderators which affect associations of the model concepts with DSM behaviours, and that the extent of moderation is based on levels of the moderator. High levels of moderators are assumed to directly influence DSM behaviours, whereas lower levels of moderators are assumed to act through the IMB-DSM model constructs to influence DSM behaviours and will not obscure the relationships between these constructs and DSM behaviours. These moderators include depressive symptom, female gender and educational level.

Depressive symptoms have been consistently found to negatively affect DSM behaviours, including SMBG, diabetes foot care, diet modification, managing high or low blood glucose, and overall DSM performance in these studies. ${ }^{62-44}$ Generally, female patients had better overall DSM performance than their male counterparts. ${ }^{13}{ }^{45}$ People with a higher educational level tended to manage their diabetes better, compared with people with lower educational level. ${ }^{26}{ }^{34} 46$ As extant studies investigating factors associated with DSM are predominately univariate in nature, these studies generally examine a direct relationship between specific DSM behaviours and DSM-related IMB skills. So far, no studies have been conducted to systematically evaluate how potential moderators may influence the relationship among the variables in the IMB-DSM model, and this study is expected to fill those knowledge gaps.

\section{Objectives}

In the proposed study, we will refine and longitudinally test culturally tailored IMB-DSM among 250 Chinese adults residing in Chengdu, China.

Aim 1: In Chinese adults with T2D, to determine which baseline variables are most strongly related to baseline DSM behaviours, controlling for age, gender, duration of diabetes diagnosis, differences in diabetes treatment and prior diabetes DSM education.

Research question (RQ): Among baseline DSM-related IMB skills, which is most strongly related to baseline DSM behaviours?

Aim 2: To examine the feedback loop as described in the original IMB model by investigating the relationships between baseline health outcomes (HbA1c, blood pressure, blood lipids and diabetes-dependent quality of life) and DSM-related information, motivation, behavioural skills and subsequent DSM behaviours at 3-month follow-up period, controlling for age, gender, duration of diabetes diagnosis, differences in diabetes treatment and prior diabetes DSM education at baseline.

RQ1: What is the relationship between baseline HbA1c levels and DSM-related information, motivation, behavioural skills and subsequent DSM behaviours at 3-month follow-up?

RQ2: What is the relationship between baseline blood pressure and DSM-related information, motivation, behavioural skills and subsequent DSM behaviours at 3-month follow-up? 
RQ3: What is the relationship between baseline blood lipids and DSM-related information, motivation and behavioural skills and subsequent DSM behaviours at 3-month follow-up?

RQ4: What is the relationship between baseline diabetes-dependent quality of life and DSM-related information, motivation, behavioural skills and subsequent DSM behaviours at 3-month follow-up?

Aim 3: In Chinese adults with T2D, to examine mediating and moderating factors associated with DSM behaviours at baseline and the 3-follow-up period.

RQ1: Are behavioural skills (diabetes self-efficacy and coping strategies) mediators of DSM behaviours at baseline and the 3-month follow-up period?

RQ2: Are depressive symptoms, female gender and educational level moderators of DSM behaviours at baseline and the 3 - follow-up period?

\section{METHODS AND ANALYSIS \\ Design}

A descriptive, repeated-measure design is used to examine the relationships among variables in the IMB-DSM model, the role of variables in predicting baseline DSM behaviours, the potential moderators and mediators, and investigate the relationship between baseline health outcomes and DSM-related information, motivation, behavioural skills and subsequent DSM behaviours at 3-month follow-up. Data will be collected at two time points, 3 months apart. Since HbAlc is considered the gold standard for monitoring glycaemic control and reflects a person's glucose control for the preceding $8-10$ weeks, ${ }^{10} 3$ months of observation is deemed long enough to reflect the glucose control.

\section{Sample and setting}

This study will be conducted in the Chengdu metropolitan area, which is the provincial capital of Sichuan province in South-West China. One community health centre will be selected from each of six urban districts in the Chengdu metropolitan area: the Yulin community health centre in Wuhou District, the Supo community health centre in Qingyang District, the Longzhoulu community health centre in Jinjiang District, the Caojiaxiang community health centre in Jinniu District, the Shuanglin community health centre in Chenghua District, and the Guixi community health centre in Gaoxin District. The inclusion criteria for participation in the research are: (A) Diagnosed with T2D. (B) Able to read, write and speak Chinese. (C) At least 18 years of age. (D) A score of the Chinese version of Mini-Mental State Examination (C-MMSE) >24 (see Screening below). (E) no other chronic physical or mental disorders. (F) Mentally competent to give informed consent. Patients will be excluded if they are pregnant, have been diagnosed with cancer or organ failure, refuse to participate in the proposed study, or have self-identified bilateral hearing loss or cognitive impairment (inability to comprehend the informed consent).

\section{Sample size}

The power analysis and sample size software was used for sample size calculation. Sample size was calculated based on the weakest correlation among all the tested variable pairs, that is, diabetes knowledge and social support $(\mathrm{r}=0.197)$. Assuming $80 \%$ power, type I error rate of 0.05 , and attrition rate of $20 \%$, a total of 250 participants will be needed to detect the correlation coefficient of 0.197 . This sample size will have higher power to detect correlations with a larger coefficient.

\section{Participant recruitment}

The research team will actively recruit participants from six selected community health centres. The principal investigator and her undergraduate mentors have established relationships with these community health centres and will be able to facilitate recruitment from these sites. An electronic medical database of the residents kept at each community health centre will also be used to identify patients with T2D in these communities. The research team will also have access to the database from which participants can be recruited.

Once approval from the Ethics Committees for Clinical Trials and Biomedical Research in the Fourth People's Hospital of Chengdu has been obtained, patients who come to the local clinics for evaluations and care will be approached after agreeing to hear about the study by a research staff member, who will explain the study, provide a consent form for review, answer questions and seek their participation. Subjects who agree to participate will be screened on inclusion criteria. Screening (see Screening below) will take about $10 \mathrm{~min}$. Those who meet study criteria will be entered into the study. In addition, advertisements about the study will be placed on buses and subways. Those interested in the study will be invited to the study site and asked to participate after reviewing study procedures and consent forms. Weekly and monthly recruitment goals will be set to ensure adequate progress on participant enrolment. If recruitment is slow, recruitment procedures will be reviewed, problems identified and adjustments will be made so that participant accrual is conducted at a satisfactory rate.

\section{Screening}

A questionnaire will be used to assess participants' eligibility with questions on age, length of diabetes, period of time when starting diabetes treatment, provider referral and willingness to participate. Since this population is at risk for cognitive impairment that may, in some cases, limit their capacity to provide consent, ${ }^{47}$ the C-MMSE will be administered to evaluate global cognitive functioning of all eligible participants. The C-MMSE has been used among Chinese adults with T2D. ${ }^{48}$ Those who obtain a score of up to 23 will be excluded because subjects 
with such low scores were considered to have possible dementia. ${ }^{49}$ Potential participants with serious cognitive problems will be referred immediately to a mental health professional used by the respective community health centres.

\section{Reducing attrition}

The proposed study will face a potential problem with attrition because the 3-month follow-up for this longitudinal study design opens up to the problem of attrition. Steps that will be taken to reduce attrition include: informing subjects of the importance of continued participation in all aspects of the study; giving a subject incentive of $¥ 155$ (about \$25) Walmart gift card per completed data collection session; making telephone calls and sending a reminder card prior to scheduled data collections. A participant locator form will be completed for each subject entered in the study and at all data collection sessions, to ensure that proper contact information for the follow-up visit is maintained.

\section{Data collection and procedures}

After consenting to participate, each subject will be requested to come to the study site for baseline data collection in a private office at the research site. Baseline data collection will include administration of the following questionnaires: Demographic Data Form, the Diabetes Knowledge Questionnaire, the Health Education Form, the Provider-Patient Communication Scale, the Health Belief Scale, the Social Support Rating Scale, the Diabetes Self-efficacy Scale, the Medical Coping Modes Questionnaire, the Diabetes Self-Care Scale, the Self-rating Depression Scale and the Audit of Diabetes-Dependent Quality-of-Life. At the 3-month follow-up data collections, all the measures will be administered again except the Demographic Data Form. Data collection from each participant should take about $60 \mathrm{~min}$. The instruments will be administered by a trained research assistant as an interview to reduce respondent burden and to standardise the approach due to the differing response formats. If the participant becomes fatigued, the battery of questionnaires may be completed in two sessions.

\section{Training of data collectors}

In an effort to improve inter-rater reliability, data collectors will be trained to collect data for baseline and follow-up data collections. They will attend intensive training sessions at the beginning of the project, and periodic refresher sessions will be offered every 2-3 weeks to reinforce the basic training. Data collectors will practise conducting data collections and blood pressure measurement and these sessions will be monitored and each data collector will be certified. To control for measurement error, a data collection protocol manual will be developed that will include data collection protocol related specifically to each questionnaire and blood pressure measurement. The specific measures, including instruments and physical biomarkers, that will be used in this study are described below.

\section{Instruments}

\section{Demographics}

This questionnaire is designed to collect background data from participants on their demographic characteristics, including age, gender, income, education, marital status, length of diabetes, health insurance, current treatment plan for diabetes and relevant health history indicators.

\section{The Diabetes Knowledge Questionnaire}

Diabetes knowledge will be measured by the Chinese version of the Diabetes Knowledge Scale, which consists of 14 items. Participants will be scored on correct answers they provide in the scale. The total score ranges from 0 to 14 , with a higher score corresponding to a higher level of diabetes knowledge. Cronbach's $\alpha$ for the scale was. 62 . The score was significantly higher in a group with more education $(\mathrm{t}(28)=2.83, \mathrm{p}<0.01)$, suggesting that the Chinese version had satisfactory construct validity. ${ }^{50}$

\section{The health education form}

Information on health education in figure 1 is based on self-reports. All participants will be asked if they have had received any form of diabetes education. If the answer is yes, the patient should answer the source of diabetes education. The source of diabetes education could be healthcare professionals, community consulting service, journals/books, TV/radio, internet and others.

\section{The provider-patient communication scale}

Provider-patient communication in figure 1 will be measured by the Provider-Patient Communication Scale. The 5-item scale is scored on a Likert Scale ranging from 0 to 6 , where 0 indicates 'strongly disagree' and 6 indicates 'strongly agree'. High scores indicate a person who is confident in their capability to communicate with healthcare professionals and knows means to get access to healthcare service in order to meet their needs. The Cronbach's $\alpha$ coefficient is reported to be 0.929 , indicating excellent internal consistency. ${ }^{12}$

\section{The health belief scale}

Health beliefs (ie, personal motivation in figure 1) refer to one's perceptions about T2D and how it can be treated. ${ }^{51}$ The Health Belief Scale was developed based on the Health Belief Model by Yamei Chen. ${ }^{51}$ The 20-item scale comprises five subscales (perceived susceptibility, perceived benefits, perceived severity, perceived barriers and cues to action) and uses a 5-point Likert scale ranging from 1 (strongly disagree) to 5 (strongly agree). A higher score represents stronger health beliefs. The content validity index is 0.81 , the test-retest reliability ranges from 0.78 to 0.82 , and the Cronbach's $\alpha$ is $0.79 .{ }^{51}$ 
The social support rating scale

Social support (ie, social motivation in figure 1) will be measured by the Social Support Rating Scale. ${ }^{52}$ The 10-item instrument measures aspects of objective social support (three items), subjective social support (four items) and usage of social support (three items). The item scores range from 14 (worst possible social support) to 66 (best possible social support). ${ }^{52}$ The test-retest reliability coefficient and the internal consistency of the scale are 0.92 and $0.88-0.94$, respectively. ${ }^{34}$

\section{The diabetes self-efficacy scale}

Diabetes self-efficacy will be measured with the 7-item Chinese version of the Diabetes Self-Efficacy Scale, which evaluates how confident participants are while performing DSM behaviours. The items are scored on a 5-point Likert-type Scale, with a higher score corresponding to a higher self-efficacy in performing DSM behaviours. The Chinese version of the Diabetes Self-Efficacy Scale has a Cronbach's $\alpha$ of 0.87 . Factor analysis showed that seven items were loaded on five factors, which explains $97.9 \%$ of the variance, and the five factors indicated confidence in performing five DSM behaviours. ${ }^{50}$

\section{The medical coping modes questionnaire}

Coping strategies in figure 1 will be measured by the 20-item Chinese version of the Medical Coping Modes Questionnaire. It assesses three forms of coping strategies associated with chronic illness: confrontation, avoidance and acceptance-resignation. ${ }^{53}$ Items are answered on a 4-point Likert Scale ranging from 1 (never) to 4 (very often). Scores from the three subscales are compared, and the highest score indicates the corresponding dominant pattern of coping used by the participant. The Cronbach's $\alpha$ for the confrontation, avoidance and acceptance-resignation subscales is reported at $0.69,0.60$, 0.76 , respectively. ${ }^{53}$

\section{The diabetes self-care scale}

DSM in figure 1 will be measured by the 26-item Diabetes Self-Care Scale, which comprises six subscales: diet modification, taking medications, SMBG, foot care, physical activity, and regulating highs and lows in blood glucose. Responses for each item range from 1 (never) to 5 (very often). Total score can range from 26 to 130 by adding up scores of six subscales, with higher scores indicating better overall DSM performance. The construct validity is 0.68 , and Cronbach's $\alpha$ is $0.87 .^{54}$

\section{The self-rating depression scale}

Depressive symptoms in figure 1 will be assessed using the Self-Rating Depression Scale, a 20-item self-administered questionnaire. ${ }^{55}$ Each item is rated on a 4-point Likert Scale, ranging from 1 (very seldom) to 4 (most of the time) and computed as an original score, then multiplied by 1.25 to get the standard score. A score ranging from 53 to 62 indicates mild depressive symptoms, a score ranging from 63 to 72 indicates moderate depressive symptoms and a score higher than 72 indicates severe depressive symptoms. ${ }^{56}$

\section{The audit of diabetes-dependent quality of life}

Diabetes-related quality of life in figure 1 will be measured by the Chinese version of Audit of Diabetes-Dependent Quality of Life, which measures both generic and diabetes-specific quality of life. The first two overview items assess generic quality of life on a 7-point Likert Scale (-3 (extremely bad) to 3 (excellent)) and quality of life without diabetes. A 5-point scale $(-3$ to +1$)$ evaluates the influence of diabetes by asking participants how they would like to rate their quality of life if they did not live with diabetes. The subsequent 19 items evaluate diabetes-specific quality of life. A 5-point scale $(-3$ to +1$)$ evaluates the influence of diabetes by asking participants what specific aspects of their life would be if they did not live with diabetes. The importance of each aspect on their life is scored on a 4-point scale (0 to 3 ). The two ratings are then multiplied and summed for a final impact score which ranges from -9 to 3 , with more negative scores suggesting worse quality of life. A score of 0 is assigned to 'unimportant' domains, regardless of the magnitude of its impact. Similarly, a score of 0 is assigned to items with no impact of diabetes, regardless of their importance to quality of life. The average-weighted rating score is obtained by dividing the sum of weighted ratings for applicable domains by the number of the applicable domains. Strong reliability (Cronbach's $\alpha=0.941$ ) has been reported ${ }^{57}$ Factor analysis showed that all items had high performance in the structural validity evaluation, with most factor loading values being larger than 0.40 (varied from 0.44 to 0.88 ).$^{57}$

\section{Physical markers}

Physical markers include HbAlc, blood pressure and blood lipids. All blood samples will be processed at the Clinical Laboratory of the Fourth People's Hospital of Chengdu. After collection, the fresh venous blood samples will be immediately transported at a temperature of $4^{\circ} \mathrm{C}$ to the Clinical Laboratory within 2 hours. The samples will then be placed in a deep freezer and stored at $-80^{\circ} \mathrm{C}$ until assays start.

\section{$\mathrm{HbA1C}$}

HbA1c will be used as a marker of T2D and glycaemic control and will be analysed from whole blood samples $(4 \mathrm{~mL})$. Analysis will be conducted using high-performance liquid chromatography, with standardisation through commercially available assays (coefficient of variation $<2 \%$ ).

\section{Blood pressure}

Blood pressure will be obtained by a trained nurse using a standard sphygmomanometer for a minimum of two consecutive readings at intervals of at least $1 \mathrm{~min}$, based on the American Heart Association guidelines. ${ }^{58}$ The seated participant should have his/her back supported 
and the upper arm bared, with legs uncrossed and feet on the ground. The arm should be placed in the cuff at the heart level. The mercury column should be deflated at $2-3 \mathrm{~mm} / \mathrm{s}$, and the first and last audible sounds should be taken as systolic and diastolic pressures. Both the participant and the nurse must remain still and silent during the procedure for the most accurate reading. The average of those readings will be used to represent the patient's blood pressure.

\section{Blood lipids}

Blood lipids include TC, HDL-C, LDL-C and triglycerides. Analysis of blood lipids will be conducted using enzymatic colorimetric test, with standardisation through commercially available assays (coefficient of variation $<2 \%$ ). Fasting serum samples will be collected in the morning after 8 hours fasting.

\section{Patient and public involvement}

The development of the RQ and outcome measures was informed by previously published research studies that engaged patients' experience. We did not involve patients in the study design, recruitment and conduct of the study. Both the baseline and 3-month outcomes on blood pressure, HbA1c and blood lipids will be mailed to each participant. After we finish the data analysis, we will present the study findings in lay terms to study participants who were enrolled in our study.

\section{Data analyses}

Data analyses for Aim 1: Linear regression models will be applied to assess the associations between baseline DSM behaviours and baseline DSM-related information variables, motivation variables and behavioural skills variables, respectively, controlling for age, gender, differences in diabetes treatment, duration of diabetes diagnosis and prior diabetes DSM education at baseline.

Data analyses for Aim 2: Linear regression models will be applied to assess the associations between baseline health outcomes (ie, HbA1c, blood pressure, blood lipids, diabetes-dependent quality of life) and DSM-related information, motivation, behavioural skills, and DSM behaviours at 3-month follow-up, respectively, controlling for age, gender, differences in diabetes treatment, duration of diabetes diagnosis and prior diabetes DSM education at baseline.

Data analyses for Aim 3: Both baseline and 3-month follow-up measures will be used for Aim 3. Structural equation modelling will be used to explore the fit of the data to the IMB-DSM model. Data will be first assessed whether they meet the assumptions of maximum likelihood estimation of structural equation modelling. Then, hypotheses on structural relations among the IMB-DSM model concepts will be assessed with an analysis of the magnitude and direction of direct effects as well as indirect effects, which indicate mediation. Significant indirect effects occur when the association of a predictor with an outcome results from the predictor being linked to a third variable. The model will be evaluated using goodness-of-fit statistic $\left(\chi^{2}\right)$, the Comparative Fit Index (CFI), the root mean square error of approximation (RMSEA) and the standardised root mean residual (SRMR). A statistically non-significant $\chi^{2}(p>0.05)$ indicates a good fit between the data and the hypothesised model. A high CFI value $(\geq 0.95)$, a low SRMR value $(\leq 0.08)$, and a low RMSEA value close to $0(<0.05)$ are desirable. ${ }^{59}$

Moderators will be tested by doing multigroup testing in Analysis of a moment structures (AMOS 23). Consistent with a prior similar study, ${ }^{14}$ to examine whether depressive symptoms, female gender and educational level moderate the relationships in the model, multigroup analysis will be used to compare if there is a significant difference between a constrained model (ie, a model in which the coefficients are set equally across the groups) and an unconstrained model (ie, a model in which these coefficients are allowed to vary freely). A significant difference between these two models indicates significant differences among the groups. A number of nested models will be tested to see where the differences are.

\section{Ethics and dissemination}

The study poses little to no risk to participants and their families. Signed informed consent will be obtained from all participating families. Participation in the study does not interfere with the usual care patients receive in the primary care settings.

\section{Author affiliations}

${ }^{1}$ University of Arkansas Eleanor Mann School of Nursing, Fayetteville, AR, USA ${ }^{2}$ Department of Nursing, The Clinical Hospital of Chengdu Brain Science Institute, MOE Key Lab for Neuroinformation, University of Electronic Science and Technology of China, Chengdu, Sichuan Province, China

${ }^{3}$ UT Health San Antonio School of Nursing, San Antonio, TX, USA

${ }^{4}$ Department of Epidemiology and Biostatistics, University of Georgia College of Public Health, Athens, GA, USA

${ }^{5}$ University of Utah College of Nursing, Salt Lake City, UT, USA

${ }^{6}$ Department of Natural Sciences/Nursing, University of Houston-Downtown, Houston, TX, USA

${ }^{7}$ Department of Biostatistics, Tulane University School of Public Health and Tropical Medicine, New Orleans, LA, USA

${ }^{8}$ National Clinical Research Center for Geriatrics, West China Hospital, Sichuan University, Chengdu, Sichuan Province, China

Acknowledgements The authors would gratefully acknowledge the assistance of Constance Johnson, PhD, MS, RN, FAAN, professor and associate dean for research and scholarship at University of Texas Health Science Center at Houston Cizik School of Nursing, in reviewing the draft of this protocol.

Contributors All authors have contributed to the conception and design of this study. TL, CL and DW drafted the manuscript. JW, RY, SG, YD and YW revised the manuscript. All authors approved the final version to be submitted to the journal.

Funding This work was supported by the Science and Technology Project of the Health Planning Committee of Sichuan under Grant No.17PJ086 to DW.

Competing interests None declared.

Patient consent Not required.

Provenance and peer review Not commissioned; externally peer reviewed.

Open access This is an open access article distributed in accordance with the Creative Commons Attribution Non Commercial (CC BY-NC 4.0) license, which 
permits others to distribute, remix, adapt, build upon this work non-commercially, and license their derivative works on different terms, provided the original work is properly cited, appropriate credit is given, any changes made indicated, and the use is non-commercial. See: http://creativecommons.org/licenses/by-nc/4.0/.

\section{REFERENCES}

1. International Diabetes Federation. IDF diabetes atlet. 8th edn. India: International Diabetes Federation, 2017. (accessed 28 Nov 2017).

2. $\mathrm{Xu} \mathrm{Y}$, Wang L, He J, et al. Prevalence and control of diabetes in Chinese adults. JAMA 2013;310:948-59.

3. Centers for Disease Control and Prevention (CDC). Awareness of prediabetes-United States, 2005-2010. MMWR Morb Mortal Wkly Rep 2013;62:209-12.

4. Anderson RM, Funnell MM, Butler PM, et al. Patient empowerment. Results of a randomized controlled trial. Diabetes Care 1995;18:943-9.

5. Glasgow RE, Anderson RM. In diabetes care, moving from compliance to adherence is not enough. Something entirely different is needed. Diabetes Care 1999;22:2090-2.

6. Luo X, Liu T, Yuan X, et al. Factors influencing self-management in Chinese adults with type 2 diabetes: a systematic review and metaanalysis. Int J Environ Res Public Health 2015;12:11304-27.

7. Shrivastava SR, Shrivastava PS, Ramasamy J. Role of self-care in management of diabetes mellitus. J Diabetes Metab Disord 2013;12:14

8. Norris SL, Lau J, Smith SJ, et al. Self-management education for adults with type 2 diabetes: a meta-analysis of the effect on glycemic control. Diabetes Care 2002;25:1159-71.

9. Cochran J, Conn VS. Meta-analysis of quality of life outcomes following diabetes self-management training. Diabetes Educ 2008;34:815-23

10. American Diabetes Association. 4. LiFestyle management: standards of medical care in diabetes-2018. Diabetes Care 2018;41:S38-S50.

11. Sun $X$, Huang $X$, Yuan $C$, et al. The influence of health belief and coping style on self-management behavior in patients with type 2 diabetes mellitus [Chinese]. Journal of Nursing 2012;19(1A):8-11.

12. Gao J, Wang J, Zhu Y, et al. Validation of an information-motivationbehavioral skills model of self-care among Chinese adults with type 2 diabetes. BMC Public Health 2013;13:100.

13. Yu P. The relationship between coping strategy, health beliefs and self-management among people with type 2 diabetes in Changsha [Master thesis]. Central South University 2009.

14. Kelly S, Melnyk BM, Belyea M. Predicting physical activity and fruit and vegetable intake in adolescents: a test of the information, motivation, behavioral skills model. Res Nurs Health 2012;35:146-63.

15. Fisher WA, Fisher JD, Harman J. The information-moticationbehavioral slills model: A general social psychological approach to understanding and promoting health behavior. In: Suls J, Wallston $\mathrm{KA}$, eds. Social psychological foundations of health and illness. Malden, MA: Wiley-Blackwell, 2003:82-106.

16. Fisher JD, Fisher WA. Changing AIDS-risk behavior. Psychol Bull 1992;111:455-74.

17. Fisher JD, Fisher WA, Amico KR, et al. An information-motivationbehavioral skills model of adherence to antiretroviral therapy. Health Psychol 2006;25:462-73.

18. Huang J, Liu Y, Zhang Y, et al. Correlation between self-management and knowledge of and attitude to diabetes in type 2 diabetic patients in Changsha [Chinese]. Journal of Central South University 2013;38:176-81.

19. Kong L, Ye Y, Ding F, et al. The relationship between diabetes knowledge, self-efficaicy, and self-management practice among individuals with type 2 diabetes in community [Chinese]. Journal of Hubei University of Science and Technology 2013;27:334-8.

20. Wang X, Lv W, Ding Y, et al. Study on correlation between selfmanagement behaviors of elderly type 2 diabetic patients and diabetes knowledge level [Chinese]. Chinese Nursing Research 2013;27:2973-4.

21. Quan J. The research about the current status and influencing factors of self-management behavior in middle-aged and over patients who with type 2 diabetes mellitus [Master thesis]. Yanbian University 2013.

22. Wang X, Lan W, Liu J, et al. Evaluation analysis of influencing factors of type II diabetes patients self-management in Shanxi Province [Chinese]. Journal of Qiqihar Medical College 2012;33:1065-9.

23. $\mathrm{Hu}$ J, Gruber KJ, Liu H, et al. Diabetes knowledge among older adults with diabetes in Beijing, China. J Clin Nurs 2013:22:51-60.

24. $\mathrm{Xu} \mathrm{Y,} \mathrm{Toobert} \mathrm{D,} \mathrm{Savage} \mathrm{C,} \mathrm{et} \mathrm{al.} \mathrm{Factors} \mathrm{influencing} \mathrm{diabetes}$ self-management in Chinese people with type 2 diabetes. Res Nurs Health 2008;31:613-25.
25. Jia Y, Wang J, Liu W. Study on self-care level of patients with type 2 diabetes mellitus and analysis of related factors [Chinese]. Nursing Journal of Chinese People's Liberation Army 2005;22:21-3.

26. Meng F. Research on health education and self-managing level of community non-insulin dependent diabetic patients [Master thesis] Sun Yat-Sen University 2010.

27. Wang L, Zhang J. Self-management of elderly patients with type 2 diabetes and its influencing factors [Chinese]. Journal of Nursing 2012;19(4A):39-42.

28. Guo XH, Yuan L, Lou QQ, et al. A nationwide survey of diabetes education, self-management and glycemic control in patients with type 2 diabetes in China. Chin Med J 2012;125:4175-80.

29. Wang J, Zhang X. Survey of self-care behavior among 2 type diabetes patients [Chinese]. Journal of Nurses Training 2002;17:663-5.

30. Gao J, Wang J, Zheng P, et al. Effects of self-care, self-efficacy, social support on glycemic control in adults with type 2 diabetes. BMC Fam Pract 2013;14:66.

31. Osborn CY, Egede LE. Validation of an information-motivationbehavioral skills model of diabetes self-care (IMB-DSC). Patient Educ Couns 2010;79:49-54.

32. Sun X, Huang X, Yuan C, et al. The relationship between coping strategy and self-management behavior among patients with type 2 diabetes [Chinese]. Journal of Nurses Training 2012;27:1084-6.

33. Du L, Lin T, Liu X. Study on correlation between social support and self-care behavior of type 2 diabetes mellitus patients [Chinese]. Chinese Nursing Research 2012;388:697-9.

34. Huang M, Zhao R, Li S, et al. Self-management behavior in patients with type 2 diabetes: a cross-sectional survey in western urban China. PLoS One 2014;9:e95138.

35. Wan $Q$, Shang S, Liu Y, et al. A study of factors of the self-care behaviors of the elderly with type 2 diabetes [Chinese]. Chinese General Practice 2003;6:659-62.

36. Fang S, Yu Z, Tao Y. The relationship between coping strategy, self-efficacy, and diabetes self-management among people living in rural areas [Chinese]. World Health Digest Medical Periodical 2013;10:79-80.

37. Gao H. The relationship between diabetes self-efficacy and self-care behavior in patients with type 2 diabetes [Chinese]. Jilin Medical Journal 2012;33:7646-7.

38. Jia $Y$, Gong T, Sang M. Investigating on self-management and selfefficacy of patients with type 2 diabetes mellitus [Chinese]. Modern Nursing 2005;11:1586-8.

39. Wang JQ, Tak-Ying Shiu A. Diabetes self-efficacy and self-care behaviour of Chinese patients living in Shanghai. J Clin Nurs 2004;13:771-2.

40. Wan $Q$, Wang $Q$, Shang S. Correlation of self-management behavior and coping patterns of patients with type 2 diabetes [Chinese]. Nursing Journal of Chinese People's Liberation Army 2008;25(1B):18-21.

41. Sundaram M, Kavookjian J, Patrick JH, et al. Quality of life, health status and clinical outcomes in Type 2 diabetes patients. Qual Life Res 2007;16:165-77.

42. Jia $Y$, Wang J, Teng $X$. Study on the correlation between psychological health level and self-management in patients with type 2 diabetes mellitus [Chinese]. Shanghai Nursing 2004;4:1-3.

43. Lin T, Lin X, Wan L. A study on correlation between self-care behavior and psychological concordance and depression of type 2 diabetes mellitus patients [Chinese]. Chinese Nursing Research 2009;23(1A):22-4.

44. Wang $Q$, Wan $Q$, Shang S. Correlation of self-management behavior and depression in patients with type 2 diabetes [Chinese]. Journal of Nursing Science 2009;24:14-15

45. Lin T, Wang J, Qin X. Study on self-care behaviors and its related factors in patients with type 2 diabetes [Chinese]. Chinese Journal of Modern Nursing 2008;14:1254-6.

46. Xia Z, Yang T. Diabetes self-management behaviors and related factors among patients with type 2 diabetes in Xiuying District, Haikou [Chinese]. Seek Medical and Ask the Medicine 2011;9:526-7.

47. Cheng $\mathrm{G}$, Huang $\mathrm{C}$, Deng $\mathrm{H}$, et al. Diabetes as a risk factor for dementia and mild cognitive impairment: a meta-analysis of longitudinal studies. Intern Med J 2012;42:484-91.

48. Chen Y, Liu Z, Zhang J, et al. Altered brain activation patterns under different working memory loads in patients with type 2 diabetes. Diabetes Care 2014;37:3157-63.

49. Zhang M. Users' guide for psychiatric scales. Changsha, China: Human Sciences \& Technology Publishing House, 2003.

50. Xu Y, Savage C, Toobert D, et al. Adaptation and testing of instruments to measure diabetes self-management in people with type 2 diabetes in mainland China. J Transcult Nurs 2008;19:234-42. 
51. Chen Y. Study on compliance and influence factors of the rehospitalized patients with diabetes mellitus. Master thesis]. Central South Universtiy 2007.

52. Yao S, Wu D. Psychological health rating scale. Yao S, Psychological assessment Beijing, China: People's medical publishing house. edn, 2008:89-126.

53. Shen X, Jiang Q. Report on application of Chinese version of MCMQ in 701 patients [Chinese]. Chinese Journal of Behavioral Medican Science 2000;9:18-20.

54. Wang JS, Wang RH, Lin CC. Self-care behavior and related factors in outpatients newly diagnosed with non-insulin-dependent diabetes mellitus [Chinese]. Journal of Nursing 1998;45:60-74.

55. Zung WW. A self-rating depression scale. Arch Gen Psychiatry 1965;12:63-70.
56. Tang P, Psychology M. People's Medical Publishing House. Beijing, China, 2009.

57. Kong D, Ding Y, Zuo X, et al. Adaptation of the audit of diabetesdependent quality of life questionnaire to people with diabetes in China. Diabetes Res Clin Pract 2011;94:45-52.

58. Pickering TG, Hall JE, Appel LJ, et al. Recommendations for blood pressure measurement in humans and experimental animals: part 1: blood pressure measurement in humans: a statement for professionals from the subcommittee of professional and public education of the american heart association council on high blood pressure research. Circulation 2005;111:697-716.

59. Kline RB. Principles and practices of structural equation modeling. 3 ed. New York, NY: The Guilford Press, 2010. 